\title{
Aortic stiffening and its impact on left atrial volumes and function in patients after successful coarctation repair: a multiparametric cardiovascular magnetic resonance study
}

Inga Voges ${ }^{1} \mathbb{D}$, Julian Kees ${ }^{1}$, Michael Jerosch-Herold ${ }^{2}$, Hannes Gottschalk ${ }^{3}$, Jens Trentmann ${ }^{3}$, Christopher Hart ${ }^{1}$,

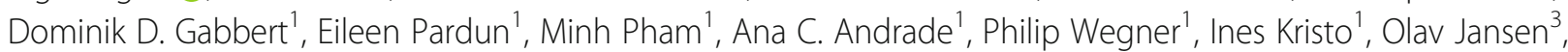
Hans-Heiner Kramer ${ }^{1}$ and Carsten Rickers ${ }^{1 *}$

\begin{abstract}
Background: The increased cardiovascular morbidity of adults with late repair of aortic coarctation (COA) has been well documented. In contrast, successful CoA repair in early childhood has a generally good prognosis, though adverse vascular and ventricular characteristics may be abnormal, which could increase long-term risk. This study sought to perform a comprehensive analysis of aortic elasticity and left ventricular (LV) function in patients with aortic coarctation (COA) using cardiovascular magnetic resonance (CMR). In a subgroup of patients, we assessed structure and function of the common carotid arteries to probe for signs of systemic vascular remodeling.

Methods: Fifty-one patients (median age 17.3 years), $13.9 \pm 7.5$ years after CoA repair, and 54 controls (median age 19.8 years) underwent CMR.

We determined distensibility and pulse wave velocity (PWV) at different aortic locations. In a subgroup, common carotid artery distensibility, PWV, wall thickness and wall area were measured. LV ejection fraction (EF), volumes, and mass were measured from short axis views. Left atrial (LA) volumes and functional parameters (LAEF Passiver $L A E F_{\text {Contractile, }} L A E F_{\text {Reservoir }}$ ) were assessed from axial cine images.

Results: In patients distensibility of the whole thoracic aorta was reduced $(p<0.05)$ while PW was only significantly higher in the aortic arch $(p<0.01)$. Distensibility of the descending aorta at the level of the pulmonary arteries and PWV in the descending aorta, both correlated negatively with age at COA repair. LA volume before atrial contraction and minimal LA volume were higher in patients $(p<0.05)$. LAEF Passive and LAEF Reservoir were reduced $(p<0.05)$, and $\operatorname{LAEF}_{\text {Reservoir }}$ correlated negatively with aortic arch PWV $(p<0.05)$. LVEF, volumes and mass were not different from controls. Carotid wall thickness and PWW were higher in patients compared to controls $(p<0.05)$.
\end{abstract}

Conclusions: Patients after CoA repair have impaired bioelastic properties of the thoracic aorta with impact on LV diastolic function. Reduced descending aortic elasticity is associated with older age at time of CoA repair. The remodeling of the common carotid artery in our sub-study suggests systemic vessel wall changes.

Keywords: Aortic coarctation, Left ventricular diastolic function, Pulse wave velocity, Aortic distensibility, Arterial stiffness

\footnotetext{
* Correspondence: rickers@pedcard.uni-kiel.de

${ }^{1}$ Department of Congenital Heart Disease and Paediatric Cardiology,

University Hospital of Schleswig-Holstein, Campus Kiel, Arnold-Heller-Str. 3,

Haus 9, 24105 Kiel, Germany

Full list of author information is available at the end of the article
} 


\section{Background}

Despite successful surgical repair, patients with aortic coarctation (CoA) have a higher cardiovascular morbidity and mortality compared to the healthy population [1, 2]. Early vascular changes have been found [3-5] that may lead to adverse left ventricular (LV) remodeling and even dysfunction. Adult CoA patients have been shown to have poor echocardiographic LV long axis function, which was related to older age at time of intervention and increased aortic stiffness [6]. Cardiovascular magnetic resonance (CMR) has revealed that $L V$ mass was elevated in young CoA patients, which was associated with impaired aortic bioelasticity [7].

CMR has become an important imaging tool in the longterm follow-up of CoA patients because it is well validated for measuring ventricular volumes, mass and parameters describing systolic function, and it has the ability to perform high resolution imaging of the cardiovascular anatomy in 3-dimensional space [8, 9]. Recently, it has been shown that CMR can provide quantitative data on left atrial (LA) volumes which can be used as markers of LV diastolic function $[10,11]$. Furthermore, CMR allows accurate assessment of regional vascular distensibility, pulse wave velocity (PWV) [12-14], as well as structural vascular changes, for instance in the carotid arteries $[15,16]$.

For the current study, we hypothesized that in CoA patients after surgical repair the thoracic aorta shows increased stiffness which impacts the LV function. In a sub-study, we wanted to determine whether CMR can detect systemic changes of vascular bioelasticity and structure, and focused for this on the common carotid artery, the major source of cerebral vascular supply.

\section{Methods}

\section{Patients}

Fifty-one patients after surgical repair were consecutively recruited during follow-up at our institution. Exclusion criteria were evidence of a more than mild re-CoA (mean gradient $>20 \mathrm{mmHg}$ ), mitral valve stenosis (mean gradient $>8 \mathrm{mmHg}$ ) and more than mild aortic or mitral valve regurgitation, all assessed by echocardiography.

In patients and controls at least three blood pressure (BP) measurements were taken at the time of CMR. In children blood pressure percentiles were calculated using the fourth report from the National High Blood Pressure Education Program, Working Group on Children and Adolescents from the US National Institutes of Health, USA [17]. In adults ( $\geq 18$ years) blood pressure was classified using the 2013 guidelines from the task force for the management of arterial hypertension of the European Society of Hypertension and of the European Society of Cardiology [18]:

1) Children (<18 years): Stage 1 hypertension: systolic and/or diastolic BP ranging from $95^{\text {th }}$ to $99^{\text {th }}$ percentile plus $5 \mathrm{mmHg}$. Stage 2 hypertension: systolic and/or diastolic $\mathrm{BP}>99^{\text {th }}$ percentile plus $5 \mathrm{mmHg}$.

2) Adults ( $\geq 18$ years): Stage 1 hypertension: systolic BP ranging from 140 to $159 \mathrm{mmHg}$ and/or diastolic BP ranging from 90 to $99 \mathrm{mmHg}$. Stage 2 hypertension: systolic BP ranging from 160 to $179 \mathrm{mmHg}$ and/or diastolic BP ranging from 100 to $109 \mathrm{mmHg}$.

For comparison to patients, heart-healthy, agematched controls were recruited among outpatients, medical students, and hospital staff.

In CoA patients $<8$ years, sedation with propofol and midazolame was used for the CMR study. Monitoring of electrocardiogram, BP and oxygen saturation using a CMR compatible monitoring system (Precess ${ }^{\mathrm{TM}}$, Invivo, Florida, USA) was performed in patients and controls.

Written informed consent was obtained from all patients, controls, parents or guardians, as appropriate. The study protocol was approved by the ethics committee of the medical faculty of the Christian Albrechts University in Kiel (Nr. A104/10).

\section{CMR acquisition}

CMR studies were done with a 3.0-Tesla scanner (Achieva 3.0 T, Philips Medical Systems, Netherlands) with a phased-array coil (SENSE ${ }^{\mathrm{TM}}$ Cardiac coil, Philips Medical Systems, Netherlands). In a subgroup of 11 patients and 13 controls, common carotid artery imaging was performed using an 18-element head and neck coil (SENSE Neurovascular coil 18 elements, Philips Medical Systems, Netherlands). This head and neck coil was available only for the last patients enrolled $(n=11)$.

Gradient echo cine CMR with retrospective gating was applied to measure aortic cross-sectional areas (CSA), to describe dimensions at end-systole and end-diastole, and to analyse aortic distensibility. The angulation of the images slices was adjusted to locally intersect the axis of the aorta at, or close to $\left(\sim \pm 10^{\circ}\right)$ a right angle (as we used stacks of parallel slices for sections of the ascending and descending aorta to speed up scan planning and acquisition, it was not possible to intersect the aorta in all slices at exactly a right angle). The scan parameters were as follows: Field of view (FOV) $280 \times 224 \mathrm{~mm}$, matrix size 149 $\times 116$, voxel size $1.88 \times 1.94 \times 6 \mathrm{~mm}, \mathrm{TR} / \mathrm{TE}=4.4 / 2.5 \mathrm{~ms}$, 25 cardiac phases, number of repetitions: 2 , scan duration per slice: $15 \mathrm{~s}$. To assess LA volumes and functional parameters indicating LV diastolic function we applied axial gradient-echo cine sequences with retrospective ECG gating $[10,11]$. The sequence parameters were as follows: FOV $280 \times 224 \mathrm{~mm}$, matrix size $149 \times 116$, voxel size 1.88 $\times 1.94 \times 6 \mathrm{~mm}, \mathrm{TR} / \mathrm{TE}=4.4 / 2.5 \mathrm{~ms}, 25$ cardiac phases, number of repetitions: 2 , scan duration per slice: $15 \mathrm{~s}$.

To evaluate aortic PWV we performed two-dimensional phase-contrast (PC) imaging with through-plane velocity 
encoding (VENC $=2 \mathrm{~m} / \mathrm{s}$ ) and retrospective ECG gating for three locations in the ascending (AAo) and descending aorta (DAo; parameters: FOV $270 \times 270 \mathrm{~mm}$, matrix size $165 \times 193$, voxel size $1.64 \times 1.4 \times 7 \mathrm{~mm}, \mathrm{TR} / \mathrm{TE}=4.4 /$ $2.7 \mathrm{~ms}$, velocity encoding strength $200 \mathrm{~cm} / \mathrm{s}, 80$ phases, kspace segmentation factor of 1 , SENSE factor 1.8, scan duration 45-75 s). The maximal temporal resolution corresponded to $2 \times$ repetition time, equaling $9 \mathrm{~ms}$. The first image plane allowed simultaneous flow measurement in the AAo and the proximal DAo using a slice plane intersecting the aorta at both locations at an approximately right angle. The second image plane was perpendicular to the DAo at the level of the diaphragm.

LV volumes and systolic function were measured using a gradient-echo cine sequence in the short-axis plane. Imaging parameters included the following: FOV $330 \times$ $330 \mathrm{~mm}$, matrix size $176 \times 190$, voxel size $1.88 \times 1.74 \times$ $6 \mathrm{~mm}, \mathrm{TR} / \mathrm{TE}=3.7 / 1.8 \mathrm{~ms}, 25$ cardiac phases, number of repetitions: 2, scan duration: 3-6 min.

Gradient-echo cine imaging of the neck with retrospective ECG gating was used to measure carotid CSA areas as a basis for calculating distensibility. The parameters were: FOV $280 \times 224 \mathrm{~mm}$, matrix size $149 \times 116$, voxel size 1.88 $\times 1.94 \times 6 \mathrm{~mm}, \mathrm{TR} / \mathrm{TE}=4.4 / 2.5 \mathrm{~ms}, 25$ cardiac phases, number of repetitions 2, scan duration 3-6 min.

For the estimation of carotid artery PWV, flow was measured in the proximal and distal (just below the bifurcation) carotid artery by using a retrospectively-gated PC sequence with the following parameters: FOV $270 \times 270$ $\mathrm{mm}$, matrix size $165 \times 193$,voxel size $1.64 \times 1.4 \times 7 \mathrm{~mm}$, $\mathrm{TR} / \mathrm{TE}=4.4 / 2.7 \mathrm{~ms}$, velocity encoding strength $200 \mathrm{~cm} / \mathrm{s}$, 80 phases, $\mathrm{k}$-space segmentation factor of 1 , SENSE factor 1.8 , scan duration 45-75 $\mathrm{s}$; maximal temporal resolution corresponded to $2 \times$ repetition time, equaling $9 \mathrm{~ms}$.

Carotid arterial wall thickness and wall area were assessed by using a multislice T2 dark-blood fast spinecho sequence (parameters: FOV $160 \times 160 \mathrm{~mm}$, matrix size $251 \times 384$, voxel size $0.2 \mathrm{~mm}$, slice thickness $2 \mathrm{~mm}$, $\mathrm{TR} / \mathrm{TE}=3000 / 80 \mathrm{~ms}$ ).

\section{CMR data analysis}

Image analysis was performed with software for cardiac analysis (Extended MR WorkSpace 2.6.3.2 HF3, Philips Medical Systems, Netherlands).

Aortic CSA's were measured from oblique or double oblique cine images at four locations of the thoracic aorta at the time of the minimal and maximal distension of the cardiac cycle. The measuring points were the aortic root, the AAo, the DAo at the level of the pulmonary arteries (aortic isthmus) and the DAo at the diaphragm (Fig. 1). Carotid CSA were measured at the proximal and distal carotid artery. CSA were used to describe aortic anatomy and dimensions as well as to evaluate aortic and carotid distensibility.

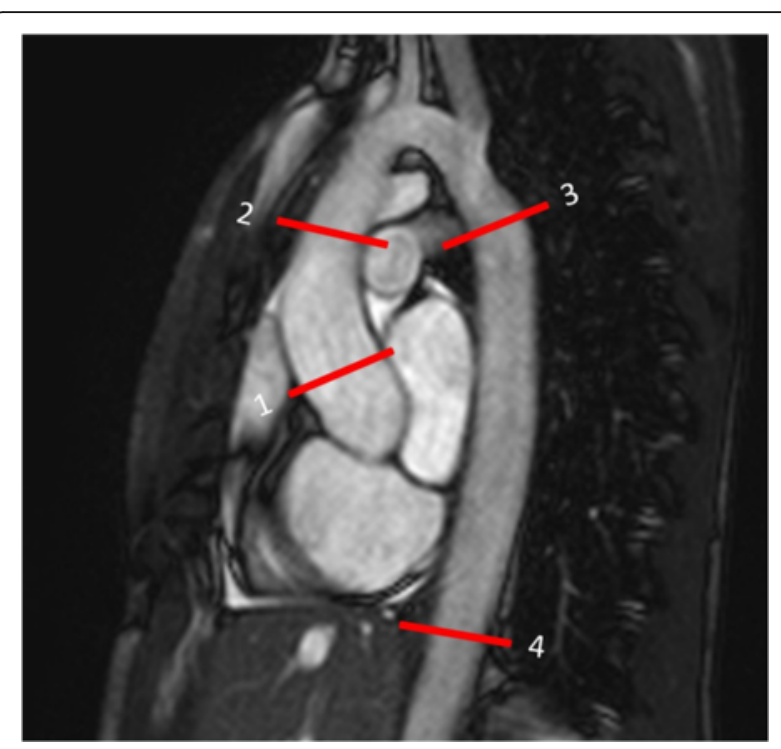

Fig. 1 Gradient-echo cine images show assessment of aortic CSA. CSA were measured at four levels: 1) aortic root, 2) AAo, 3) DAo at the pulmonary bifurcation and 4) DAo at the diaphragm

Distensibility was calculated according to the following formula [19]:

$$
\left(\mathrm{A}_{\max }-\mathrm{A}_{\min }\right) /\left(\mathrm{A}_{\min } *\left(\mathrm{P}_{\min }-\mathrm{P}_{\max }\right)\right)
$$

where $A_{\min }$ is the minimal and $A_{\max }$ is the maximal CSA. $\mathrm{P}_{\min }$ and $\mathrm{P}_{\max }$ are the systolic and diastolic BP. BP were recorded with a sphygmomanometer during CMR. The cuff was placed around the right upper arm.

PWV was assessed from PC measurements in two predefined aortic segments. The first segment extended from the AAo to the DAo at the level of the pulmonary arteries, the second segment corresponded to a section of the DAo from the level of the pulmonary arteries to the diaphragm. Furthermore, we measured common carotid artery PWV. Flow versus time curves from $\mathrm{PC}$ cine images were obtained and the time delay ( $\Delta \mathrm{t}$, Fig. 2a) of the distal flow curve relative to the proximal flow curve was determined by a validated method, which is based on the crosscorrelation between the systolic up-stroke portions of two flow waveforms [20]. The midline distance between the particular positions was measured on angulated sagittal images $(\Delta x$, Fig. $2 b)$ and for the carotid artery on images from the time-of-flight angiography. PWV was then calculated by the means of the following equation:

$$
\operatorname{PWV}(\mathrm{m} / \mathrm{s})=\Delta \mathrm{x} / \Delta \mathrm{t} .
$$

LV end-diastolic volume (LVEDV), LV end-systolic volume (LVESV) and LV mass were obtained from short axis cine images by drawing endocardial and epicardial contours at end diastole and end systole. LV stroke volume (SV) was calculated as the difference of LVEDV 


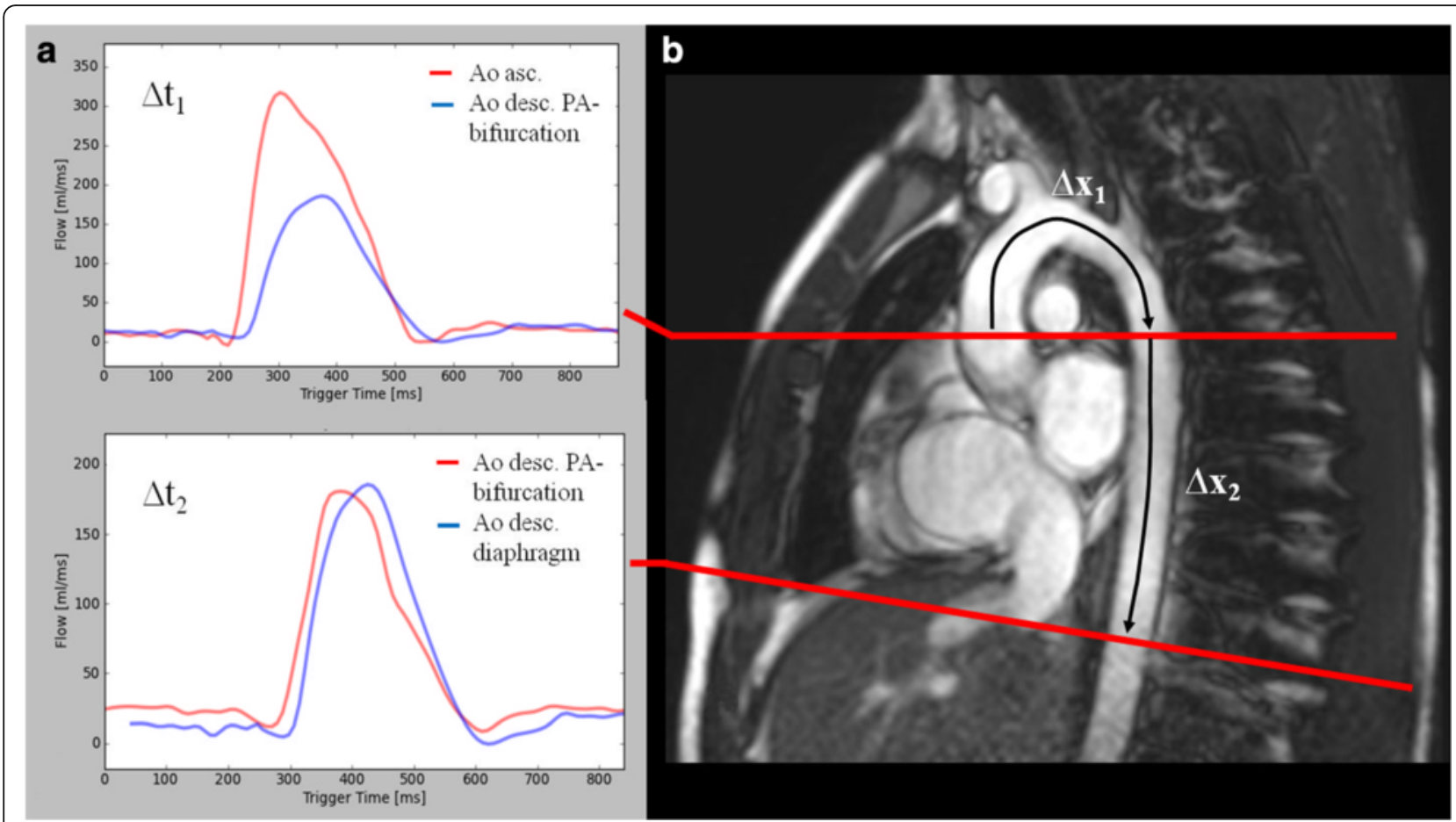

Fig. 2 a Aortic flow-curves from PC cine imaging. We used the validated method cross-correlation to determine the time delay ( $\Delta t$ ) between the proximal and distal flow curves [20]. b Sagittal angulated cine image which shows the orientation of scan planes for PC imaging. The distance between the particular positions $(\Delta t)$ was measured by drawing a curved-line following the midline course of the thoracic aorta

from the LVESV, and LV ejection fraction (LVEF) was obtained by dividing the LVSV by the LVEDV.

LA volumes were quantified using Simpson's rule and manual planimetry of axial cine images [15]. Tracings were performed at different times in the cardiac cycle (Fig. 3): 1) maximal LA volume before mitral valve opening $\left.\left(\mathrm{LA}-\mathrm{Vol}_{\max }\right), 2\right)$ before LA contraction (LA-Vol ${ }_{\mathrm{ac}}$ ) and 3) minimal LA volume at mitral valve closure (LA$\left.\mathrm{Vol}_{\text {min }}\right)$. We used this dimensions to measure additional LA volumes and functional parameters [11, 21]:

LA total emptying volume $=\mathrm{LA}-\mathrm{Vol}_{\max }-\mathrm{LA}-\mathrm{Vol}_{\min }$ LA passive emptying volume $\left(\mathrm{V}_{\text {Passive }}\right)=\mathrm{LA}-\mathrm{Vol}_{\max }-$ LA-Vol ${ }_{\mathrm{ac}}$

LA contractile volume $\left(\mathrm{V}_{\text {Contractile }}\right)=\mathrm{LA}-\mathrm{Vol}_{\mathrm{ac}}-\mathrm{LA}-$ $\mathrm{Vol}_{\text {min }}$

LA passive emptying function $\left(\mathrm{LAEF}_{\text {Passive }}\right)=(\mathrm{LA}-$

$\left.\mathrm{Vol}_{\max }-\mathrm{LA}-\mathrm{Vol}_{\mathrm{ac}}\right) * 100 \% / \mathrm{LA}-\mathrm{Vol}_{\max }$,

LA contractile emptying function ( $\left.\mathrm{LAEF}_{\text {Contractile }}\right)$

$=\left(\mathrm{LA}-\mathrm{Vol}_{\mathrm{ac}}-\mathrm{LA}_{\mathrm{Vol}} \mathrm{Vol}_{\mathrm{min}}\right) * 100 \% / \mathrm{LA}-\mathrm{Vol}_{\mathrm{ac}}$,

LA reservoir emptying function $\left(\mathrm{LAEF}_{\text {Reservoir }}\right)=(\mathrm{LA}-$

$\left.\mathrm{Vol}_{\max }-\mathrm{LA}-\mathrm{Vol}_{\min }\right)^{*} 100 \% / \mathrm{LA}-\mathrm{Vol}_{\max }$.

LV and LA volumes were indexed to body surface area (BSA).

Carotid wall thickness was measured at two positions and two sites for each carotid artery, respectively [16].
Wall area was assessed by manual tracing of the inner and outer contour of the carotid wall on the T2 darkblood fast spinecho images as reported previously [15] and shown in Fig. 4.

\section{Statistical analysis}

Statistical analysis was performed using MedCalc Version 12.3.0.0 (MedCalc statistical software, Mariakerke, Belgium) and R (R Foundation for Statistical Computing, Vienna, Austria; URL: http://www.R-project.org/).

To determine if a variable was normally distributed we visually assessed a quantile-quantile plot of the data. All variables except age appeared on the Q-Q plots to be normally distributed, with deviations from the ideal distribution at the tails that were relatively small. All continuous variables were expressed either as mean \pm standard deviation if they appeared to be normally distributed, or otherwise as median with range. Differences between patients and controls were compared using the Mann-Whitney-U test. Correlations between variables were measured by Spearman's rank method. All tests were two-tailed. $P$ values of less than 0.05 were considered to indicate statistical significance. Linear regression analysis for distensibility at four aortic locations was performed with linear mixed-effects (LME) methods to account for any intrapatient correlation of distensibility measurements. The model for distensibility included a random intercept, age 


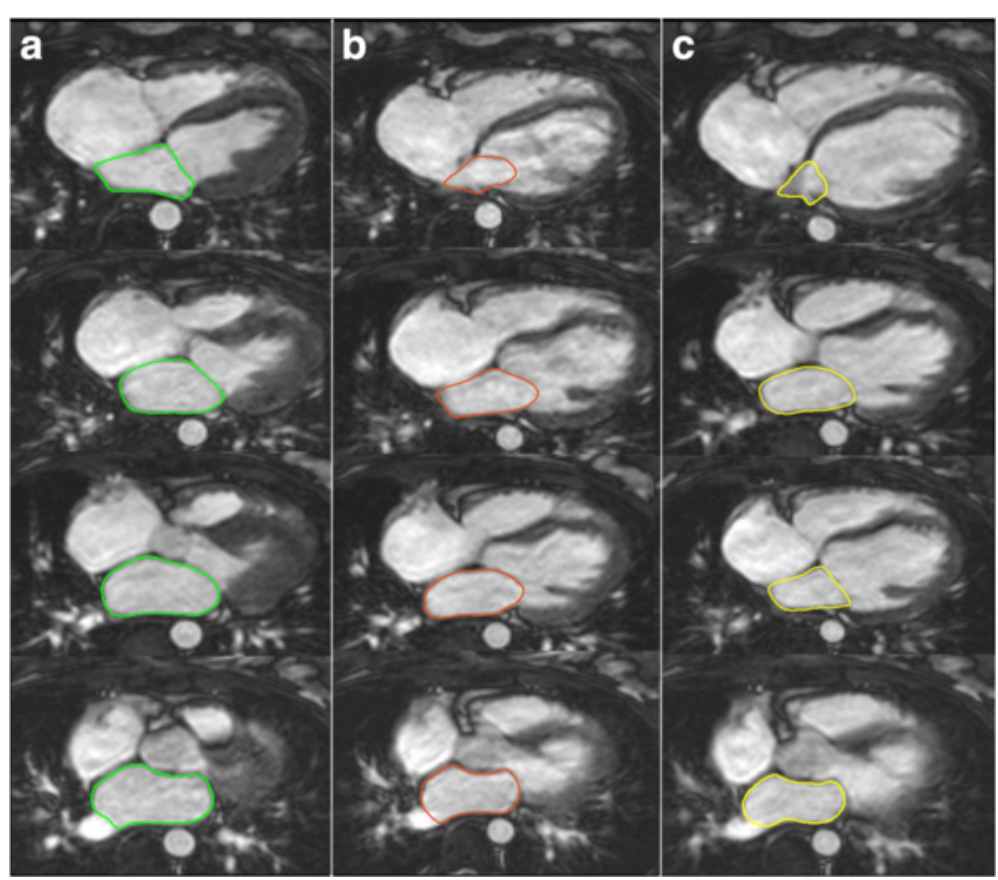

Fig. 3 Volumetric assessment of LA volumes on axial cine images: a LA-Vol max (green). $\mathbf{b} L A-V o l_{a c}(r e d)$ and $\mathbf{c} L A-V o I_{\min }$ (yellow)

at the time of repair, age at time of MRI, and measurement location. For the measurement location with 4 levels (root, ascending aorta, isthmus, and descending aorta) we used a so-called "treatment" contrast matrix, where the descending aorta, which was least affected by CoA, served as reference level. LME analysis was performed with the "lme4" package in R(version 1.1-12; URL: http://cran.rproject.org).

\section{Results}

51 patients (median 17.3 years; $0.9-42.3$ years) with CoA (median age at repair 1.0 years; 0.01-28.1 years) were

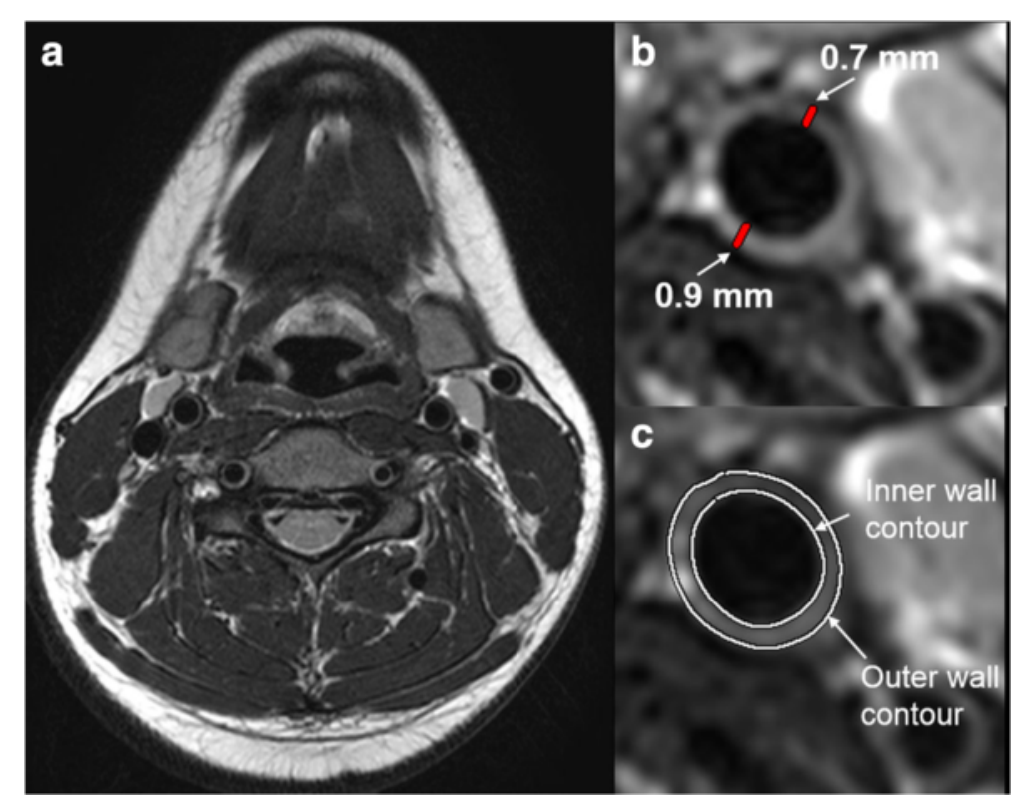

Fig. 4 Measurement of carotid wall thickness and area from (a) T2 dark-blood fast spinecho images. Wall thickness (b) was measured at two positions for each vessel. Wall area (c) was determined by drawing an outer and inner contour to measure first the entire vessel area (including the vessel wall) and the lumen area. The lumen area was then subtracted from the vessel area [15] 
recruited for the study. 27 patients were younger than 18 years and 24 patients were 18 years or older. Of the 27 patients, only 6 were younger than 10 years. 26 patients underwent surgery before the age of 1 year (median 0.04 years; $0.01-0.89$ years) and 25 patients were older than 1 year at surgery (median 6.5 years; $1.2-28.1$ years). 54 individuals served as healthy controls. A previous study from our group in healthy volunteers showed relatively small changes for aortic PWV between the ages of 2 and 28 years [22]. For this reason, we enrolled patients with ages covering a relatively broad range to maximize the chances of detecting an association of PWV and other bioelastic properties with the age at CMR, and distinguish this from any association with age at the time of CoA repair.

Six patients needed interventional (balloon dilatation $n=5$, stent implantation $n=1)$ treatment of re-CoA at a median time difference of $0.4(0.5-14)$ years after surgery. Two patients underwent reoperation, one of them after unsuccessful balloon angioplasty. Sixteen patients had a bicuspid aortic valve without significant stenosis or insufficiency. In seven patients a ventricular septal defect was closed surgically. In addition, there were 3 patients with a small ventricular septal defect and one with a partial anomalous pulmonary venous connection. None of the patients had evidence for re-CoA or an aortic aneurysm shown by CMR during the study.

34 patients had normal BP, 4 patients had stage 1 hypertension and 13 patients needed antihypertensive treatment which was effective at the time of the study. Mean and diastolic BP were not different between patients and controls.
Characteristics of patients and controls are summarized in Table 1.

\section{Regional aortic dimensions and bioelasticity}

There were no significant differences in aortic CSA between patients and controls. Patients with a bicuspid aortic valve had an enlarged CSA of the AAo compared to age-matched patients without a bicuspid aortic valve $\left(439.1 \pm 101.1\right.$ vs. $\left.332.5 \pm 88.5 \mathrm{~mm}^{2} / \mathrm{m}^{2}, p=0.007\right)$. The presence of a bicuspid aortic valve had no effect on aortic distensibility or PWV.

In patients distensibility was significantly lower than in controls at all positions of the thoracic aorta (Table 2). In CoA patients aortic root distensibility was lowest $(p=0.05)$ and trended lower in the aortic isthmus $(p=0.07)$, compared to the descending aorta (Fig. 5). Furthermore, distensibility across the different locations was lower if the repair was performed at a later age $(p=0.016$; Fig. 5$)$. As patients who had aortic repair later, also tended to be older, both age at time of repair and age at time of CMR were used as predictors in the model for distensibility. Age at the time of MRI did not have a significant effect.

Aortic arch PWV was significantly elevated in patients compared to controls, whereas PWV in the DAo was not significantly different between patients and controls (Table 2), and this difference remained significant with simultaneous adjustment by age. In controls PWV in the DAo trended higher compared to the aortic arch $(+0.45 \mathrm{~m} / \mathrm{s} ; p=0.07)$, but in patients, PWV in the DAo was significantly lower than in the aortic arch $(-0.82 \mathrm{~m} /$

Table 1 Clinical characteristics of CoA patients and controls

\begin{tabular}{|c|c|c|c|}
\hline Characteristic & Patients $(n=51)$ & Controls $(n=54)$ & $P$ value \\
\hline Age (y) & $17.3(0.9-42.3)$ & $19.8(2.3-40.1)$ & 0.54 \\
\hline Age at initial surgery $(y)$ & $4.2 \pm 6.1$ & NA & NA \\
\hline Female/Male (n) & $18 / 33$ & $31 / 23$ & NA \\
\hline Weight (kg) & $61.8 \pm 26.6$ & $58.0 \pm 21.3$ & 0.35 \\
\hline Height (cm) & $163.7 \pm 24.2$ & $165.5 \pm 20.9$ & 0.98 \\
\hline $\mathrm{BSA}\left(\mathrm{m}^{2}\right)$ & $1.7 \pm 0.5$ & $1.6 \pm 0.5$ & 0.36 \\
\hline Systolic blood pressure $(\mathrm{mmHg})^{a}$ & $111.9 \pm 15.4$ & $107.0 \pm 8.7$ & 0.047 \\
\hline Diastolic blood pressure $(\mathrm{mmHg})^{a}$ & $60.5 \pm 9.6$ & $61.6 \pm 10.5$ & 0.79 \\
\hline Mean blood pressure $(\mathrm{mmHg})^{\text {a }}$ & $80.1 \pm 10.7$ & $79.1 \pm 9.8$ & 0.35 \\
\hline Pulse Pressure $(\mathrm{mmHg})^{\text {a }}$ & $51.5 \pm 14.3$ & $45.3 \pm 9.0$ & 0.04 \\
\hline Heart rate $(\mathrm{bpm})^{\mathrm{a}}$ & $74.1 \pm 15.7$ & $70.3 \pm 16.9$ & 0.38 \\
\hline \multicolumn{4}{|l|}{ Medications (n) } \\
\hline Beta-blockers & 7 & - & - \\
\hline ACE inhibitors & 6 & - & - \\
\hline Diuretics & 3 & - & - \\
\hline
\end{tabular}

Data are presented as mean \pm SD or median and range. $P$-Values are from the Mann-Whitney- $U$ test $A C E$ angiotensin-converting enzyme, $B S A$ body surface area

${ }^{a}$ Data were measured at the time of the CMR study 
Table 2 Comparison of CMR measurements in patients and controls

\begin{tabular}{|c|c|c|c|}
\hline Variable & Patients $(n=51)$ & Controls $(n=54)$ & $p$ Value \\
\hline \multicolumn{4}{|l|}{ Maximal aortic area $\left(\mathrm{mm}^{2} / \mathrm{m}^{2}\right)$} \\
\hline Aortic root & $449.5 \pm 126.1$ & $440.5 \pm 93.0$ & 0.95 \\
\hline Ascending aorta & $353.2 \pm 104.5$ & $356.3 \pm 68.4$ & 0.41 \\
\hline Descending aorta at the isthmus & $163.2 \pm 61.5$ & $176.9 \pm 32.0$ & 0.02 \\
\hline Descending aorta at the level of the diaphragm & $151.9 \pm 35.3$ & $155.2 \pm 38.0$ & 0.82 \\
\hline LVEDV $\left(\mathrm{ml} / \mathrm{m}^{2}\right)$ & $81.0 \pm 15.3$ & $80.1 \pm 12.0$ & 0.91 \\
\hline $\operatorname{LVESV}\left(\mathrm{ml} / \mathrm{m}^{2}\right)$ & $30.4 \pm 12.0$ & $31.0 \pm 6.2$ & 0.28 \\
\hline $\operatorname{LVSV}\left(\mathrm{ml} / \mathrm{m}^{2}\right)$ & $50.6 \pm 8.2$ & $49.0 \pm 7.9$ & 0.35 \\
\hline LVEF (\%) & $63.3 \pm 8.0$ & $61.3 \pm 4.4$ & 0.04 \\
\hline LV mass $\left(\mathrm{g} / \mathrm{m}^{2}\right)$ & $60.7 \pm 14.1$ & $57.5 \pm 14.2$ & 0.25 \\
\hline LA Vol $\max \left(\mathrm{ml} / \mathrm{m}^{2}\right)$ & $47.5 \pm 10.2$ & $43.2 \pm 8.7$ & 0.07 \\
\hline LA Vol ${ }_{\mathrm{ac}}\left(\mathrm{ml} / \mathrm{m}^{2}\right)$ & $32.3 \pm 8.0$ & $27.4 \pm 5.9$ & $<0.01$ \\
\hline LA Vol $\min \left(\mathrm{ml} / \mathrm{m}^{2}\right)$ & $24.6 \pm 6.1$ & $20.9 \pm 5.1$ & $<0.01$ \\
\hline LA Total emptying volume $\left(\mathrm{ml} / \mathrm{m}^{2}\right)$ & $23.0 \pm 6.1$ & $22.5 \pm 5.7$ & 0.98 \\
\hline$V_{\text {Passive }}\left(\mathrm{ml} / \mathrm{m}^{2}\right)$ & $15.3 \pm 5.0$ & $15.6 \pm 4.9$ & 0.50 \\
\hline$V_{\text {Contractile }}\left(\mathrm{ml} / \mathrm{m}^{2}\right)$ & $7.7 \pm 3.3$ & $6.5 \pm 2.7$ & 0.06 \\
\hline $\mathrm{LAEF}_{\text {Contractile }}(\%)$ & $23.7 \pm 7.2$ & $23.7 \pm 8.1$ & 1.00 \\
\hline LAEF $_{\text {Passive }}(\%)$ & $32.2 \pm 8.1$ & $36.9 \pm 6.6$ & $<0.01$ \\
\hline $\operatorname{LAEF}_{\text {Reservoir }}(\%)$ & $48.4 \pm 6.9$ & $51.9 \pm 6.8$ & $<0.05$ \\
\hline \multicolumn{4}{|l|}{ Distensibility $\left(10^{-3} \mathrm{mmHg}^{-1}\right)$} \\
\hline Aortic root & $5.6 \pm 3.8$ & $7.4 \pm 3.0$ & $<0.01$ \\
\hline Ascending aorta & $5.8 \pm 3.1$ & $8.1 \pm 3.6$ & $<0.01$ \\
\hline Descending aorta at the isthmus & $5.7 \pm 3.0$ & $6.8 \pm 2.3$ & $<0.01$ \\
\hline Descending aorta at the level of the diaphragm & $6.8 \pm 2.8$ & $8.0 \pm 2.8$ & $<0.05$ \\
\hline PWV aortic arch (m/s) & $4.6 \pm 1.7$ & $3.5 \pm 0.8$ & $<0.01$ \\
\hline PWV descending aorta $(\mathrm{m} / \mathrm{s})$ & $4.3 \pm 1.6$ & $3.9 \pm 0.8$ & 0.70 \\
\hline
\end{tabular}

Data are presented as mean $\pm \mathrm{SD}$. P-Values are from the Mann-Whitney-U test

LVEF left ventricular ejection fraction, $L V S V$ left ventricular stroke volume, $L V E D V$ left ventricular end-diastolic volume, $L V E S V$ left ventricular end-systolic volume, $L A$ $\mathrm{Vol}_{\text {max }}$ maximal left atrial volume, $L A V_{\text {Ool }}$ min minimal left atrial volume, $L A V_{\text {Ooc }}$ left atrial volume just before atrial contraction, $L A E F_{\text {contractile }}$ left atrial contractiale emptying function, $L A E F_{\text {Passive }}$ left atrial passive emptying function, $L A E F_{\text {Reservoir }}$ left atrial reservoir emptying function, $P W V$ pulse wave velocity, $V_{\text {Contractile }}$ left atrial contractile volume, $V_{\text {Passive }}$ left atrial passive emptying volume

s; $P=0.03)$, with both comparisons being made with simultaneous adjustment by age $(p<1 \mathrm{e}-5)$. PWV in the DAo correlated with age at repair $(r=0.33, p<0.05)$, consistent with the effect of age at time of repair that was observed for aortic distensibility.

\section{LV systolic and diastolic function}

LV volumes and mass indexed by BSA were similar in patients and controls (Table 2). LVEF was higher in patients. There was no correlation between reduced aortic distensibility or increased PWV and these LV parameters $(p>0.05)$.

LA-Vol $\mathrm{ac}_{\mathrm{ac}}$ and $\mathrm{LA}-\mathrm{Vol}_{\mathrm{min}}$ were significantly higher and $\mathrm{LV}-\mathrm{Vol}_{\max }$ trended higher in patients (Table 2). LA- $\mathrm{Vol}_{\mathrm{ac}}$ correlated with higher aortic arch PWV $(r=0.33, p<$ 0.05). Furthermore, $\mathrm{LAEF}_{\text {Passive }}$ and $\mathrm{LAEF}_{\text {Reservoir }}$ were significantly reduced in the patient group compared to the control group (Table 2) and $\mathrm{LAEF}_{\text {Reservoir }}$ correlated negatively with aortic arch PWV $(r=-0.35, p<0.05$, Fig. 6). In patients who underwent surgery after the age of 1 year $\operatorname{LAEF}_{\text {Passive }}$ was significantly lower compared to patients who were younger than 1 year $(29.2 \pm 8.9 \mathrm{vs}$. $34.7 \pm 6.5, p<0.05)$. LAEF $_{\text {Passive }}$ was reduced in patients with arterial hypertension compared to patients without arterial hypertension.

\section{Bioelasticity, wall thickness and wall area of the common carotid artery}

Table 3 demonstrates the comparison of carotid artery measurements between patients and controls. PWV, wall thickness and wall area were significantly higher in 


\section{Coarc. Patients}

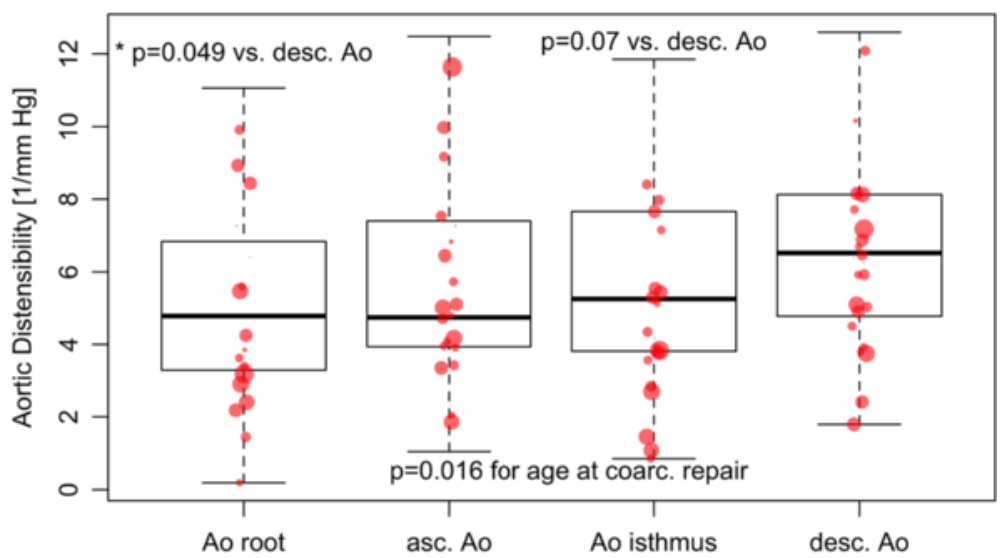

Fig. 5 In patients with repaired COA the distensibility of the aorta was significantly lower in the aortic root and trended lower in the aortic isthmus, compared to the descending aorta. Both age at time of repair and aortic location were included in a linear mixed effects model, and the $p$-values shown in the graph were obtained from this model, which accounts for repeated measurements (at 4 aortic locations) in each patient. Across all locations, the distensibility was lower if the repair was performed at a later age $(p=0.016)$. The size of the data points is proportional to the age at the time of surgery, and larger data points are seen for lower distensibility values, illustrating the significant effect of higher age at time of repair. The DAo was chosen as reference level, as the values there were closest to those observed in normal volunteers

patients than in controls. Carotid distensibility was not statistically different between both groups.

\section{Discussion}

After successful CoA repair, patients with normal LV systolic function and size, had significantly decreased distensibility of the entire thoracic aorta, and PWV was significantly higher in the aortic arch indicating adverse aortic remodeling. LA markers of left-sided diastolic dysfunction were impaired and correlated partly with the aortic bioelastic markers. Distensibility of the entire aorta was lower if CoA repair was performed at later age.

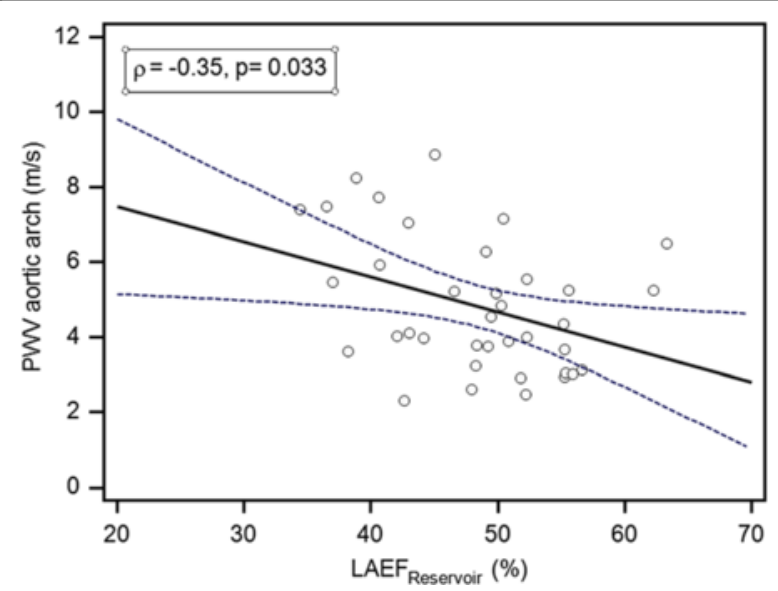

Fig. 6 Relation between $L A E F_{\text {Reservoir }}$ and aortic arch PW. The dotted lines represent the borders of the $95 \%$ confidence intervals

\section{Regional aortic bioelasticity}

In our patients distensibility was reduced in all parts of the thoracic aorta compared to controls. Consistent with this, PWV was increased in the aortic arch, but not in the DAo.

Ou et al. evaluated aortic bioelasticity in 40 normotensive CoA patients by CMR and found a reduced distensibility of the AAo but not of the DAo at the pulmonary bifurcation [7]. Two other echocardiographic studies showed an impaired AAo distensibility and increased stiffness index before CoA repair and also after successful operation but elastic properties of the abdominal DAo were not different from controls $[3,4]$. The discrepancies might be explained by difference in median patient age as our cohort was 6-18 years older compared to the cited studies. Additionally, Ou et al. excluded patients with arterial hypertension or on cardiac medication [7]. The mean BP of our patients was not different compared to our controls.

There are several reasons for a reduced aortic bioelasticity in CoA. Histopathological studies by Niwa et al. showed abnormalities of the aortic media, both proximally and distal to the coarctation [23]. The decreased elasticity of the DAo at the pulmonary bifurcation, representing the isthmic area, can additionally be caused by scar formation due to the surgical procedure. In addition, arterial hypertension leads to structural and functional arterial wall alterations [24]. In our cohort we found no differences between patients with (4 pts had stage 1 hypertension, 13 pts under effective antihypertensive treatment) and patients without arterial hypertension which supports the notion that our patients did not have severe arterial hypertension. 
Table 3 Comparison of carotid artery MRI measurements in patients and controls

\begin{tabular}{llll}
\hline Variable & Patients $(n=11)$ & Controls $(n=13)$ & \\
\hline Distensibility $\left(10^{-3} \mathrm{mmHg}^{-1}\right)$ & & & \\
$\quad$ Proximal right carotid artery & $10.4 \pm 7.0$ & $5.9 \pm 3.8$ & $0.9 \pm 3.0$ \\
Distal right carotid artery & $8.3 \pm 2.9$ & $8.1 \pm 5.5$ & 0.64 \\
Proximal left carotid artery & $8.7 \pm 5.8$ & $6.2 \pm 2.2$ & 0.36 \\
Distal left carotid artery & $7.1 \pm 4.8$ & $3.3 \pm 1.5$ & 0.74 \\
PWV right carotid artery $(\mathrm{m} / \mathrm{s})$ & $6.8 \pm 4.1$ & $4.2 \pm 1.7$ & $<.01$ \\
PWV left carotid artery $(\mathrm{m} / \mathrm{s})$ & $6.9 \pm 4.0$ & $15.3 \pm 2.6$ & $<.10$ \\
Wall area right carotid artery $\left(\mathrm{mm}^{2}\right)$ & $19.4 \pm 2.4$ & $15.8 \pm 1.9$ & $<0.01$ \\
Wall area left carotid artery $\left(\mathrm{mm}^{2}\right)$ & $19.7 \pm 4.1$ & $0.75 \pm 0.09$ & $<0.05$ \\
Wall thickness right carotid artery $(\mathrm{mm})$ & $0.90 \pm 0.12$ & $0.76 \pm 0.08$ & $<0.01$ \\
Wall thickness left carotid artery $(\mathrm{mm})$ & $0.90 \pm 0.12$ & \\
\hline
\end{tabular}

Data are presented as mean \pm SD. $P$-Values are from the Mann-Whitney-U test

Cohen et al., showed more than 25 years ago, that older age at the time of repair contributes to the risk of hypertension [2]. We found a correlation between aortic distensibility at the pulmonary bifurcation as well as PWV in the DAo and age at surgical CoA repair, respectively. The association between age at repair and DAo distensibility remained significant with simultaneous adjustment by age at time of CMR.

\section{LV systolic and diastolic function}

This is the first CMR study which evaluated the relation between aortic bioelasticity and LV systolic and diastolic function in CoA patients. LA size has been shown to be a reliable and important indicator of diastolic dysfunction and it provides prognostic information in various kinds of cardiac diseases [25-27]. In this study, we have not only used maximum LA volume but have measured also several other LA volumes and LA functional parameters to describe LA function during the cardiac cycle and to assess the relative contribution of LA function to LV filling which is dependent on LV diastolic function $[26,27]$.

We found that LA volumes, $\mathrm{LA}-\mathrm{Vol}_{\mathrm{ac}}$ and $\mathrm{LA}-\mathrm{Vol}_{\min }$, were significantly higher and that $\mathrm{LAEF}_{\text {Passive }}$ and LAE$F_{\text {Reservoir, }}$ all markers of LV diastolic function, were reduced in patients compared to controls. Increased aortic arch PWV correlated with $\mathrm{LA}-\mathrm{Vol}_{\mathrm{ac}}$ and $\mathrm{LAEF}_{\text {Reservoir }}$. Myocardial mass was similar in patients and controls, which might be explained by the fact that all but 4 patients had normal BP.

Our findings show, that CoA-patients have an impaired LV diastolic function which might result from the impaired aortic bioelastic function, which can increase LV afterload. It has been speculated that an impaired aortic bioelasticity can impair diastolic function through early reflection of the pulse wave leading to increased LV afterload and decreased coronary perfusion [28]. The increased afterload and the decreased coronary perfusion, may compromise myocardial relaxation and promote subendocardial ischemia as well as interstitial fibrosis leading to reduced LV compliance [28-30]. An echocardiographic study of CoA patients by Lombardi et al. recently also demonstrated that an elevated aortic stiffness is linked to diastolic impairment [31]. Using tissue Doppler imaging, Florianczyk et al. also found abnormal LV diastolic mechanics in patients after successful CoA repair, but did not evaluate the aortic bioelasticity [32].

Arterial hypertension is known to cause LV diastolic dysfunction in the longterm [32]. In our patients we found markers for LV diastolic dysfunction despite all but 4 having normal BP. We suppose that already early stages of increased aortic stiffness promotes LV diastolic dysfunction in CoA patients. This may have clinical implications as to avoid cardiovascular risk factors aggravating aortic stiffness.

Patients with diastolic dysfunction have an abnormal relaxation and an increased LV chamber stiffness impairing LV filling [33]. In patients with preserved LV systolic function, diastolic dysfunction is related to poor outcome [34]. The early detection of impaired aortic bioelasticity and diastolic dysfunction may therefore be important for optimal patient management.

\section{Common carotid artery bioelasticity, wall thickness and wall area}

Patients in our substudy showed an increased carotid wall thickness and area as well as a higher PWV while the distensibility as a parameter of wall stiffness was not increased. These findings do not contradict one another. Rather, they demonstrate that bioelasticity, in terms of the elastic modulus of the carotid arteries was not impaired, and the higher PWV in patients can be attributed to the increased carotid wall thickness [35, 36]. For 
comparisons of PWV's the effect of vessel wall thickness needs to be taken into account [36].

Two former ultrasound studies found a decreased carotid distensibility after CoA repair [37, 38]. An increased intima-media thickness of the carotid artery, indicating adverse vascular remodeling, has been reported by ultrasound studies [39]. Our results of increased wall thickness and area in patients are in line with these findings. They underline, that the increased arterial stiffness is not restricted to the aorta. The alterations found in the carotid arteries may be rather representative for other parts of the systemic vascular bed.

\section{Study limitations}

The present study has several limitations. Although our study included 51 patients and 54 controls, only in a subgroup of 11 patients and 13 controls the carotid artery was examined. However, as significantly different results were obtained even in these small groups, carotid wall changes must be considered as severe. As the range of BP was rather small with only a few hypertensive patients $(n=4)$ we could not analyze the effect of BP on anatomical and functional vascular changes.

\section{Conclusions}

Patients after CoA repair show reduced aortic bioelasticity of the entire thoracic aorta which was associated with older age at repair. LV diastolic function was impaired, despite normal BP in most patients, which suggests that the increase of aortic stiffness sufficiently increases the LV afterload to induce LV diastolic dysfunction. Monitoring of aortic bioelastic and LV functional parameters is therefore important during followup. In addition, our study supports the strategy to treat CoA patients expeditiously after initial diagnosis.

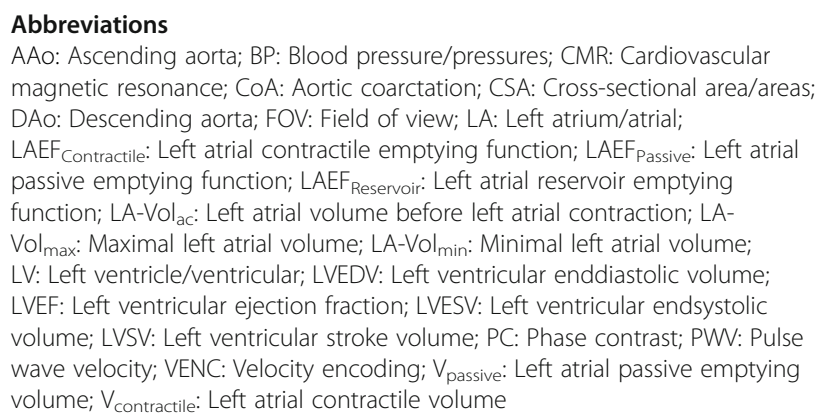

\section{Acknowledgements}

The authors thank Mrs. Traudel Hansen (CMR technologist) for her assistance with patient management.

\section{Funding}

None.

\section{Availability of data and materials}

The datasets supporting the conclusions of this article are included within the article.

\section{Authors' contributions}

IV and CR developed the concept and design of the study, collected the data and analyzed the results. They also drafted the manuscript and had the primary responsibility for the final content. HG, JT and $\mathrm{CH}$ assisted substantially in data acquisition and analysis. DDG, EP, MP, AAC, PW, OJ and IK revised the article for important intellectual content. MJH and HHK participated mainly in the design of the study and the critical revision of the manuscript. All authors have read and approved the final manuscript.

\section{Authors' information}

Not applicable.

\section{Competing interests}

The authors declare that they have no competing interests.

\section{Consent for publication}

Not applicable.

\section{Ethics approval and consent to participate}

Written informed consent was obtained from all patients, controls, parents or guardians, as appropriate. The study protocol was approved by the ethics committee of the medical faculty of the Christian Albrechts University in Kiel (Nr. A104/10).

\section{Author details}

${ }^{1}$ Department of Congenital Heart Disease and Paediatric Cardiology, University Hospital of Schleswig-Holstein, Campus Kiel, Arnold-Heller-Str. 3, Haus 9, 24105 Kiel, Germany. Department of Radiology, Brigham \& Women's Hospital and Harvard Medical School, 75 Francis Street, Boston, MA 02115, USA. ${ }^{3}$ Department of Diagnostic Radiology, University Hospital of Schleswig-Holstein, Campus Kiel, Arnold-Heller-Straße 3, 24105 Kiel, Germany.

Received: 8 August 2016 Accepted: 26 August 2016

Published online: 12 September 2016

\section{References}

1. Pedersen TA, Munk K, Andersen NH, Lundorf E, Pedersen EB, Hjortdal VE, et al. High long-term morbidity in repaired aortic coarctation: weak association with residual arch obstruction. Congenit Heart Dis. 2011;6:573-82.

2. Cohen M, Fuster V, Steele PM, Driscoll D, McGoon DC. Coarctation of the aorta. Longterm follow-up and prediction of outcome after surgical correction. Circulation. 1989;80:840-5.

3. Vogt M, Kühn A, Baumgartner D, Baumgartner C, Busch R, Kostolny M, et al. Impaired elastic properties of the ascending aorta in newborns before and early after successful coarctation repair: proof of a systemic vascular disease of the prestenotic arteries? Circulation. 2005;111:3269-73.

4. Kühn A, Baumgartner D, Baumgartner C, Hörer J, Schreiber C, Hess J, et al. Impaired elastic properties of the ascending aorta persist within the first 3 years after neonatal coarctation repair. Pediatr Cardiol. 2009:30:46-51.

5. de Divitiis M, Pilla C, Kattenhorn M, Zadinello M, Donald A, Leeson P, et al. Vascular dysfunction after repair of coarctation of the aorta: impact of early surgery. Circulation. 2001;104 Suppl 1:1165-70.

6. Lam YY, Mullen MJ, Kaya MG, Gatzoulis MA, Li W, Henein MY. Left ventricular long axis dysfunction in adults with "corrected" aortic coarctation is related to an older age at intervention and increased aortic stiffness. Heart. 2009;95:733-9.

7. Ou P, Celermajer DS, Jolivet O, Buyens F, Herment A, Sidi D, et al. Increased central aortic stiffness and left ventricular mass in normotensive young subjects after successful coarctation repair. Am Heart J. 2008;155:187-93.

8. Pennell DJ, Sechtem UP, Higgins CB, Manning WJ, Pohost GM, Rademakers FE, European Society of cardiology, Society for Cardiovascular Magnetic Resonance, et al. Clinical indications for cardiovascular magnetic resonance (CMR): consensus panel report. J Cardiovasc Magn Reson. 2004;6:727-65.

9. Bottini PB, Carr AA, Prisant LM, Flickinger FW, Allison JD, Gottdiener JS. Magnetic resonance imaging compared to echocardiography to assess left ventricular mass in the hypertensive patient. Am J Hypertens. 1995;8:221-8.

10. Sarikouch S, Koerperich H, Boethig D, Peters B, Lotz J, Gutberlet M, et al. Reference values for atrial size and function in children and young adults by cardiac MR: a study of the German competence network congenital heart defects. J Magn Reson Imaging. 2011;33:1028-39. 
11. Kaminski M, Steel K, Jerosch-Herold M, Khin M, Tsang S, Hauser T, et al. Strong cardiovascular prognostic implication of quantitative left atrial contractile function assessed by cardiac magnetic resonance imaging in patients with chronic hypertension. J Cardiovasc Magn Reson. 2011;13:42.

12. Leeson CP, Robinson M, Francis JM, Robson MD, Channon KM, Neubauer $S$, et al. Cardiovascular magnetic resonance imaging for non-invasive assessment of vascular function: validation against ultrasound. J Cardiovasc Magn Reson. 2006:8:381-7.

13. Grotenhuis HB, Westenberg JJ, Steendijk P, van der Geest RJ, Ottenkamp J, Bax JJ, et al. Validation and reproducibility of aortic pulse wave velocity as assessed with velocity-encoded MRI. J Magn Reson Imaging. 2009;30:521-6.

14. Metafratzi ZM, Efremidis SC, Skopelitou AS, de Roos A. The clinical significance of aortic compliance and its assessment with magnetic resonance imaging. J Cardiovasc Magn Reson. 2002;4:481-91.

15. Mani V, Aguiar SH, Itskovich W, Weinshelbaum KB, Postley JE, Wasenda EJ, et al. Carotid black blood MRI burden of atherosclerotic disease assessment correlates with ultrasound intima-media thickness. J Cardiovasc Magn Reson. 2006;8:529-34.

16. Harloff A, Zech T, Frydrychowicz A, Schumacher M, Schöllhorn J, Hennig J, et al. Carotid intima-media thickness and distensibility measured by MRI at 3 T versus high-resolution ultrasound. Eur Radiol. 2009;19:1470-9.

17. National High Blood Pressure Education Program Working Group on High Blood Pressure in Children and Adolescents. The fourth report on the diagnosis, evaluation, and treatment of high blood pressure in children and adolescents. Pediatrics. 2004;114:555-76.

18. Mancia G, Fagard R, Narkiewicz K, Redón J, Zanchetti A, Böhm M, Task Force Members, et al. 2013 ESH/ESC Guidelines for the management of arterial hypertension: the Task Force for the management of arterial hypertension of the European Society of Hypertension (ESH) and of the European Society of Cardiology (ESC). J Hypertens. 2013;31:1281-357.

19. Cavalcante JL, Lima JA, Redheuil A, Al-Mallah MH. Aortic stiffness: current understanding and future directions. J Am Coll Cardiol. 2011;57:1511-22.

20. Fielden SW, Fornwalt BK, Jerosch-Herold M, Eisner RL, Stillman AE, Oshinski JN. A new method for the determination of aortic pulse wave velocity using cross-correlation on 2D PCMR velocity data. J Magn Reson Imaging. 2008;27:1382-7.

21. Farzaneh-Far A, Ariyarajah V, Shenoy C, Dorval JF, Kaminski M, Curillova Z, et al. Left atrial passive emptying function during dobutamine stress MR imaging is a predictor of cardiac events in patients with suspected myocardial ischemia. JACC Cardiovasc Imaging. 2011;4:378-88.

22. Voges I, Jerosch-Herold M, Hedderich J, Pardun E, Hart C, Gabbert DD, Hansen JH, Petko C, Kramer HH, Rickers C. Normal values of aortic dimensions, distensibility, and pulse wave velocity in children and young adults: a cross-sectional study. J Cardiovasc Magn Reson. 2012;14:77.

23. Niwa K, Perloff JK, Bhuta SM, Laks H, Drinkwater DC, Child JS, et al. Structural abnormalities of great arterial walls in congenital heart disease: light and electron microscopic analyses. Circulation. 2001;103:393-400.

24. London GM, Guerin AP. Influence of arterial pulse and reflected waves on blood pressure and cardiac function. Am Heart J. 1999:138:220-4.

25 Simek CL, Feldman MD, Haber HL, Wu CC, Jayaweera AR, Kaul S. Relationship between left ventricular wall thickness and left atrial size: comparison with other measures of diastolic function. J Am Soc Echocardiogr. 1995;8:37-47.

26 Pritchett AM, Mahoney DW, Jacobsen SJ, Rodeheffer RJ, Karon BL, Redfield MM. Diastolic dysfunction and left atrial volume: a population-based study. J Am Coll Cardiol. 2005;45:87-92.

27 Prioli A, Marino P, Lanzoni L, Zardini P. Increasing degrees of left ventricular filling impairment modulate left atrial function in humans. Am J Cardiol. 1998:82:756-61.

28 Mottram PM, Haluska BA, Leano R, Carlier S, Case C, Marwick TH. Relation of arterial stiffness to diastolic dysfunction in hypertensive heart disease. Heart. 2005;91:1551-6

29 Leite-Moreira AF, Correia-Pinto J, Gillebert TC. Afterload induced changes in myocardial relaxation: a mechanism for diastolic dysfunction. Cardiovasc Res. 1999;43:344-53.

30 Mahfouz RA. Relation of coronary flow reserve and diastolic function to fractional pulse pressure in hypertensive patients. Echocardiography. 2013; 30:1084-90.

31 Lombardi KC, Northrup V, McNamara RL, Sugeng L, Weismann CG. Aortic stiffness and left ventricular diastolic function in children following early repair of aortic coarctation. Am J Cardiol. 2013;112:1828-33.
32 Florianczyk T, Werner B. Assessment of left ventricular diastolic function in children after successful repair of aortic coarctation. Clin Res Cardiol. 2011; 100:493-9.

33 Zile MR, Baicu CF, Gaasch WH. Diastolic heart failure-abnormalities in active relaxation and passive stiffness of the left ventricle. N Engl J Med. 2004;350: 1953-9.

34 Achong N, Wahi S, Marwick TH. Evolution and outcome of diastolic dysfunction. Heart. 2009;95:813-8.

35 Gosling RG, Budge MM. Terminology for describing the elastic behaviors of arteries. Hypertension. 2003:41:1180-2.

36 Voges I, Jerosch-Herold M, Wegner P, Hart C, Gabbert D, Al Bulushi A, Fischer G, Andrade AC, Pham HM, Kristo I, Kramer HH, Rickers C. Frequent dilatation of the descending aorta in children with hypoplastic left heart syndrome relates to decreased aortic arch elasticity. J Am Heart Assoc. 2015; 4:e002107.

37 Ou P, Celermajer DS, Mousseaux E, Giron A, Aggoun Y, Szezepanski I, et al. Vascular remodeling after "successful" repair of coarctation: impact of aortic arch geometry. J Am Coll Cardiol. 2007;49:883-90.

38 Brili S, Tousoulis D, Antoniades C, Aggeli C, Roubelakis A, Papathanasiu S, et al. Evidence of vascular dysfunction in young patients with successfully repaired coarctation of aorta. Atherosclerosis. 2005;182:97-103.

39 Vriend JW, de Groot E, de Waal TT, Zijta FM, Kastelein JJ, Mulder BJ. Increased carotid and femoral intima-media thickness in patients after repair of aortic coarctation: influence of early repair. Am Heart J. 2006;151:242-7. 